\title{
Single-cell RNA-sequencing of zebrafish hair cells reveals the novel genes potentially involved in hearing loss
}

\section{Fuping Qian}

Nantong University

Guanyun Wei

Nantong University

Xin Wang

Nantong University

Jie Gong

Nantong University

Chao Guo

Nantong University

Xiaoning Wang

Nantong University

Xu Zhang

Nantong University

Jinxiang Zhao

Nantong University

Cheng Wang

Nantong University

Mengting $\mathrm{Xu}$

Nantong University

Yuebo Hu

Nantong University

Yajing Gao

Nantong University

Guoli Yin

Nantong University

Jiahui Kang

Nantong University

Renjie Chai

Southeast University

Gangcai Xie 
Nantong University

Dong Liu ( $\sim$ liudongtom@gmail.com )

Nantong University https://orcid.org/0000-0002-2764-6544

\section{Research Article}

Keywords: Hair cell, scRNA-seq, zebrafish

Posted Date: January 18th, 2022

DOI: https://doi.org/10.21203/rs.3.rs-1241257/v1

License: (c) (1) This work is licensed under a Creative Commons Attribution 4.0 International License. Read Full License 


\section{Abstract}

Hair cells play key roles in hearing and balance, and hair cell loss would result in hearing loss or vestibular dysfunction. Cellular and molecular research in hair cell biology provide us a better understanding of hearing and deafness. Zebrafish, owing to their hair cell-enriched organs, have been widely applied in hair cell-related research worldwide. Similar to mammals, zebrafish have inner ear hair cells. In addition, they also have lateral line neuromast hair cells. These different hair cells vary in morphology and function. However, systematic analysis of their molecular characteristics remains lacking. In this study, we analyzed the GFP+ cells isolated from $\operatorname{Tg}(B r n 3 c$ :mGFP) larvae using single cell RNA-sequencing (scRNA-seq). Three subtypes of hair cells, namely macula hair cell (MHC), crista hair cell $(\mathrm{CHC})$ and neuromast hair cell $(\mathrm{NHC})$ were characterized and validated by whole-mount in situ hybridization analysis of marker genes. Furthermore, we carried out the morphological comparison of these hair cells. Based on the hair cell scRNA-seq data, we obtained hair cell-enriched genes pool, including hearing loss genes that have been identified in humans and novel genes potentially involved in hair cell formation and function. Two novel genes were discovered to specifically function in NHCs and MHCs, corresponding to their specific expression in NHCs and MHCs. This study allows us to understand the specific genes in hair cell subpopulations of zebrafish, which will shed light on the genetics of both human vestibular and cochlear hair cell function.

\section{Introduction}

Hair cells, getting their name from the hair-like structure on the surface of the cell body ${ }^{[1]}$, detect mechanical vibrations through the cilia. Mechanoelectrical transduction (MET) channels on the tip of stereocilia open in response to stimuli, which cause the depolarization of the hair cells ${ }^{[2,3]}$. Finally, the neurotransmitters are released into the synaptic cleft between the hair cell and auditory neuron ${ }^{[4-6]}$. Inner ear mechanosensory hair cells, including cochlear hair cells and vestibular hair cells, play crucial roles in hearing and balance in mammals. The cochlear hair cells function as receptors of acoustic vibration, and they can be divided into outer hair cells $(\mathrm{OHCs})$ and inner hair cells $(\mathrm{IHCs})^{[7]}$. As is known, there are two types of vestibular sensory epithelia: maculae and cristae. The maculae are located in the utricle and saccule, and the cristae lie at the ends of the three semicircular canals. Both of the vestibular sensory epithelia are composed of hair cells and supporting cells. The vestibular hair cells can be divided into type $\otimes$ and type $\otimes$ hair cells ${ }^{[8]}$. The macula hair cells can sense linear acceleration and gravitational equivalent, and crista hair cells can sense angular acceleration and deceleration ${ }^{[9,10]}$. In mammals, the cochlear hair cells get mechanical signals from the tectorial membrane, but the crista hair cells and macula hair cells sense the vibration from the endolymph and otolith, respectively.

As vertebrates, zebrafish have many organs similar to humans, which make them an excellent animal model for biological and medical research ${ }^{[11]}$. Zebrafish have an ear-like structure, otic vesicle, however, they do not have the cochlea. In the otic vesicle of zebrafish, there are five clusters of hair cells, three clusters of crista hair cells and two clusters of macula hair cells. In the cristae, including anterior crista 
$(A C)$, lateral crista (LC), and posterior crista (PC), hair cells put their kinocilia into the endolymph and detect the endolymph flow caused by head rotation. In the maculae, including the utricular macula (UM) and saccular macula (SM), the cilia of hair cells are in contact with the otoliths, which would produce movement if acceleration occurs. Therefore, the macula hair cells can sense linear acceleration and gravity. It has been demonstrated that the utricular macula is responsible for vestibular function ${ }^{[12]}$ and the saccular macula is the hearing organ ${ }^{[13]}$ in zebrafish. Unlike in mammals, zebrafish have neuromast hair cells in their lateral line system. These hair cells are located on the surface of the skin, and are sensors of the surrounding water, which help them to detect prey and avoid predators. Because they are easy to administer to and image, the neuromast hair cells have been widely used in hair cell related biomedical research, for example, in ototoxic drug screening.

Zebrafish model has been widely utilized in the hearing research field ${ }^{[14-19]}$. However the systemetic analysis of molecular and morphological differences among these hair cells is so far lacking. In this study, scRNA-seq was used to analyze zebrafish hair cell gene expression, and uncover the molecular differences among the different subtypes of hair cells in zebrafish.

\section{Results}

\section{Singlecell RNA-sequencing reveals different subpopulations of hair cells in zebrafish}

Hair cells play crucial roles in hearing and balance, and they are variable in morphology and function. To further distinguish the different types of hair cells from the molecular level, we used single-cell RNAsequencing to analyze the gene expression pattern. Here, the transgenic zebrafish line $\operatorname{Tg}(\mathrm{Brn} 3 \mathrm{c}: \mathrm{mGFP})$ (Figure 1A) was used as the animal model, and the zebrafish larvae at $6 \mathrm{dpf}$ were dissociated into the single cells by trypsin, and the GFP-positive cells were sorted using the fluorescence activated cell sorting (FACS) method (Figure S1). After high-throughput sequencing based on 10×Genomics system, the gene expression data was obtained. Based on Seurat ${ }^{[20]}$ analysis, the cells were divided into 21 clusters. By analyzing the gene expression pattern of the top genes in each cluster, the four clusters of cells, from the cluster $0,5,7,12$, were annotated as hair cells, and the cells from the cluster 2,3 and 10 were identified as retinal ganglion cells (RGCs). Both of the hair cells and retinal ganglion cells are GFP-expressing cells in the $\operatorname{Tg}(B r n 3 c: m G F P)$ zebrafish ${ }^{[21]}$. The rest were considered as other cells, which were GFP-negative and brought into the GFP-positive cells due to inevitable technical problems, including red blood cells, lymphocytes, muscle cells and so on (Figure 1B). The number of cells and marker genes detected in each cluster was shown (Figure 1C, D). For each cluster, the top marker genes were listed, and the expression pattern of these genes were also presented (Figure 1E).

\section{The morphological charicteristics of the three types of hair cells in zebrafish}

The typical feature of hair cells which differ from other cells is the cilia, including kinocilia and stereocilia, on the surface of the cell body. The cilia of hair cells can detect mechanical vibration by stereocilia bundle deflection, leading to the tension of the tip links, opening of the MET channels, and causing 
depolarization of hair cells. In zebrafish, except for inner ear, there are hair cells in their lateral line system, and the hair cells can be divided into three types according to their morphology and location, namely crista hair cells, macula hair cells, and neuromast hair cells (Figure 2A). The first two are both located in the inner ear, function importantly in hearing and balance, and the third ones are key component of the lateral line system, which are distributed on the surface of the skin, and help the fish to sense the environmental water. Although the cilia are the common structure of all hair cells, there are also differences among the three types of hair cells. The crista hair cells and macula hair cells have straight kinocilia, which seem inflexible, and the kinocilia of neuromast hair cells can bend themselves to detect the water movement (Figure 2B-D). For the length of kinocilia, taking the zebrafish larva of $3 \mathrm{dpf}$ as an example, the crista hair cells have the longest kinocilia, which are almost $30 \mu \mathrm{m}$ in length, and the kinocilia of macula hair cells and neuromast hair cells are approximately $10 \mu \mathrm{m}$ and $20 \mu \mathrm{m}$, respectively (Figure 2E). For the size of cell bodies, the macula hair cells are the biggest ones, and the cell bodies of the neuromast hair cells are the smallest among them (Figure 2F).

\section{Validation of the gene expression in the subtypes of hair cells using whole-mount in situ hybridization}

Through our analysis, as shown above, the cells from cluster $0,5,7,12$ were considered as hair cells. In our subsequent study, we focused on these cells, and further analysis showed that these hair cells can be divided into three subpopulations, namely macula hair cells (cluster 5 ), crista hair cells (cluster 12) and neuromast hair cells (cluster 0,7 ) (Figure 3A). We further analyzed the top marker genes expressed in the four clusters, and we found that some genes were mainly expressed in one of the clusters specifically, for example, tectb in cluster 5 , zpld $1 a$ in cluster 12, calm $1 b$ in cluster 0 and 7 (Figure 3B). Functional enrichment analysis showed that many of the genes expressed in the four clusters of cells above have hair cell-related biological function (Figure 3C-F), which indicated that these cells were hair cells. To further confirm the hair cell clustering and annotation, we performed the whole-mount in situ hybridization (WISH). As shown in Figure 3G, the cells with high tectb gene expression were mainly gathering in the cluster 5 , and the tectb gene was proved to be expressed specifically in the macula hair cells, including the utricular hair cells and saccular hair cells, by WISH. The zpld1a gene was mainly expressed in the crista hair cells, which were thought to be located in the cluster 12 according to our analysis (Figure $3 \mathrm{H}$ ), and this is consistent with previous studies ${ }^{[22]}$. The cells from the cluster 0 and 7 were considered as neuromast hair cells because of the expression patterns of their marker genes, such as calm $1 b$ (Figure 3I). As for cells from cluster 0 and 7, although they are both neuromast hair cells, they are different from each other. The cells from cluster 0 were classified to be mature neuromast hair cells, and the cluster 7 were classified to be young neuromast hair cells, according to the expression of the mature and young hair cell markers, $s 100 s^{[23]}$ and prox $1 a^{[24]}$, respectively in the two clusters (Figure S2).

On the other hand, we analyzed the marker gene of supporting cells, $k / f 1 \gamma^{25]}$, and found it was mainly expressed in the cells of cluster 1 (Figure S3), which suggests this cluster of cells are supporting cells. Likewise, we also analyzed the genes that were reported to be expressed in mantle cells, such as tnfsf10, ponzr6, pkhd1/1, fat1b, crb3b, cts12, ovgp1, cldne ${ }^{[18]}$, and found that cells expressing these genes with high level were clustering in cluster 9 (Figure S4). For cluster 14, these cells are closer to the supporting 
cells (cluster 1) in UMAP clustering (Figure 1B), however, they express some of genes that have be proved to be expressed in hair cells specifically, such as myo6b ${ }^{[26]}$, myo7aa ${ }^{[27]}$ (Figure S5), which raises a possibility that they are supporting cells that can differentiate into hair cells. Therefore, we concluded that the cells from cluster 1 and 9 were supporting cells and mantle cells, respectively, and the cells from the cluster 14 might be hair cell progenitors.

\section{The molecular properties of the three types of hair cells}

Knowing that different types of hair cells were clustering in different populations, we made a comparation among the distinguishable hair cells at the molecular level. As shown in Figure 1D, the number of marker genes detected in the cluster $0,5,7,12$ were $2352,1023,2029,928$, respectively. The two types of inner ear hair cells, macula hair cells and crista hair cells (cluster 5 and 12), share 568 genes, and 1677 marker genes were expressed in both of the mature and young neuromast hair cells (cluster 0 and 7). However, the neuromast hair cells and inner ear hair cells only share a small amount of marker genes, which reveals their differences on the gene expression level (Figure 4A). Furthermore, the top marker genes expressed in the different clusters were obviously different (Figure 4B). We also performed gene enrichment analysis through gene ontology (GO) term, and found that these different types of hair cells vary dramatically in biological process, molecular function and cellular component. For example, the neuromast hair cells have more energy metabolism-related activity, which raise the possibility that they require more energy to work compared to the inner ear hair cells (Figure 4C-E).

\section{The neuromast hair cells were enriched with MET gene expression}

As is known, the MET channels are required for functional hair cells and they are complex comprising of several components, such as TMC1, TMC2, TMIE and LHFPL5, CIB2 ${ }^{22,28-32]}$. Hair cells with functional MET channels are crucial for normal hearing and balance. In addition, the tip links play important roles in the stereocilia deflection and MET channel-gating, and it was proved to be composed of two cadherin, $\mathrm{CDH} 23$ and $\mathrm{PCDH} 15^{[33-35]}$. To determine whether these key molecules are also expressed in zebrafish hair cells and what difference there are among these different hair cells, we analyzed the expression pattern of the genes encoding these important proteins. As illustrated in Figure 5A-F, the orthologs of mammalian MET complex components were expressed in most of the neuromast hair cells, however, only a small proportion of inner ear hair cells can detect these gene expression. The genes encoding the tip link components, $c d h 23$ and $p c d h 15 a$, were expressed in a few hair cells (Figure 5G-H).

\section{The zebrafish hair cell scRNA-seq reveals potential hearing loss genes}

Hearing is one of the most important sensory functions, and it relies on functional hair cells. Healthy hair cells are the receptors of acoustic signals, therefore, genes responsible for hair cell development, survival and function are also important for normal hearing. There are 119 genes that have been identified as nonsyndromic hearing loss (NSHL) genes in humans (https://hereditaryhearingloss.org/). Among these genes, 96 human genes have orthologous genes in zebrafish (Figure 6A), which demonstrates that zebrafish are quite similar with human in terms of NSHL genes. Furthermore, 51 human NSHL genes 
have 57 orthologous genes which are expressed in zebrafish hair cells (cluster 0, 5, 7, and 12), and the detailed gene information were listed in Figure 6B. Except for the orthologs of identified human NSHL genes, there are still more than 3000 genes highly enriched in the zebrafish hair cells (Figure 6C), and some of these genes are thought to be crucial for hair cell function, and it is quite possible that they are potential hearing loss genes waiting to be confirmed in the future. In other words, this data may provide us clear direction for our scientific research in hearing loss gene identification. Under the guidance of this idea, we randomly selected some genes for further analysis, and found that these genes were specifically expressed in either or both of the zebrafish inner ear and neuromast hair cells (Figure 6D), indicating that these genes function in hair cell-related biological processes, and may even be essential for hearing function.

\section{Functional analysis of the candidate genes involved in hair cell development}

Our scRNA-seq analysis revealed genes which are specifically expressed in zebrafish hair cells, some of which are orthologs of human NSHL genes, however, there are many genes that have not been reported to play roles in hair cell development or function. To investigate the function of hair cell-enriched genes, we took the capgb and $m b$ gene as examples, which were expressed in neuromast hair cells and macula hair cells, respectively. Here, we used morpholino-mediated gene knockdown to downregulate the gene expression. As illustrated in Figure 7A, B, the capgb-morphants exhibited decreased hair cells in their lateral line neuromasts compared to the littermate control. Furthermore, the capgb-morphants had less response to the acoustic stimuli in the startle response test (Figure 7C, D), which indicated their hair cells had lost their function to some extent. Likewise, in another experiment, the $m b$ gene knockdown resulted in reduced macula hair cells (Figure 7E, F), and the $m b$-morphants showed severely abnormal balance ability in the vestibulo-ocular reflex (VOR) test (Figure 7G, H).

\section{Methods}

\section{Zebrafish embryos}

Zebrafish were maintained at $28.5^{\circ} \mathrm{C}$. Two zebrafish lines were used in the study, including the wildtype $A B$ and the transgenic line $T g(B r n 3 c: m G F P)$, which was described in the previous work ${ }^{[21]}$. All animal procedures were performed according to protocols approved by the Animal Care and Use Committee of Nantong University and were consistent with the National Institutes of Health Guide for the Care and Use of Laboratory Animals.

\section{Single-cell RNA-sequencing}

The $\operatorname{Tg}(B r n 3 c: m G F P)$ transgenic zebrafish larvae at 6 days post fertilization (dpf) were anaesthetized and then treated with $0.25 \%$ trypsin to dissociated into single cells, which were divided into GFP-positive and GFP-negative cells through the fluorescent activated cell sorter, and the RNA expressed in GFP-positive cells were then obtained and sequenced using the $10 \times$ Genomics platform. 


\section{Single cell sequencing analysis}

The basic procedure for single cell sequencing analysis were carried out as previously described ${ }^{[36]}$.

Briefly, Seurat V4.0.1 ${ }^{[20]}$ was used for the integrated analysis of the single cell sequencing data, including data filtering, data normalization, cell clustering and cluster-level marker gene identification. For data filtering, only the genes expressed in at least five cells were considered, the maximal percentage of reads from mitochondrial genes allowed was up to $10 \%$, and the number of genes detected for each cell was between the range of 200 to 5000 . For data normalization, sctransform was applied to data normalization, and the variables including percentage of mitochondrial expression, total number of UMls, and total number of genes detected were regressed out in a second non-regularized linear regression. For cell clustering, the principal component analysis (PCA) was first applied for extracting the top principal components (top 50 PCs), and then the clusters were identified through shared k-nearest neighbor graph construction and cluster modularity function optimization ( $k$.param $=20$, resolution $=0.25)$. For marker gene finding, Wilcoxon rank-sum test was used for comparing the target cluster to other clusters, and the $p$ values were further adjusted based on Bonferroni correction.

For $\mathrm{GO}$ enrichment analysis, the R package clusterProfiler ${ }^{[37]}$ was used, and the marker genes identified by Seurat were used as input. The three GO categories: BP (Biological Process), CC (Cellular Component), and MF (Molecular Function) were analyzed respectively against the marker genes for each cell cluster, and the $p$ values were corrected by Benjamini \& Hochberg $(\mathrm{BH})$ method, where the significantly enriched $\mathrm{GO}$ terms were defined as the ones with $\mathrm{BH}$ adjusted $\mathrm{p}$ value less than 0.05 . The $\mathrm{GO}$ enrichment results were further visualized through the enrichplot method integrated in clusterProfiler.

\section{Whole-mount in situ hybridization}

The whole-mount in situ hybridization procedure was similar to our previous description ${ }^{[38,39]}$. In brief, the gene-specific primers targeting the coding sequence of interest were designed and synthesized. After amplification using PCR method, the fragments were subcloned into the PGEM-T Easy vector (Promega). The DIG-labeling RNA probe was transcribed in vitro using the linearized recombinant plasmid as template. For the hybridization step, the pretreated zebrafish embryos were incubated with the digoxigenin-labeling RNA probe overnight firstly, and then alkaline phosphatase-conjugated primary antibody against digoxigenin was used to detect the RNA probe following three washes. Subsequently, for color reaction, the nonspecific binding was washed out and the substrate of alkaline phosphatase, NBT/BCIP solution, was added to the reaction system. Finally, the samples were imaged and the genespecific mRNA expression was visualized through microscope. The primers used for probe synthesis are as following:

tectb-F: GCCGTGTTTAGCCAAAGAGT;

tectb-R: ACAACTTCTCCCCGTCACAT;

zpld1a-F: CCACGGTTGTGCTGTTTAGC; 
zpld1a-R: GTTGGTGGCTTTTACAGCGG;

calm1b-F: AAGTGTTGTGTCGTGGGGTT;

calm1b-R: GCAGGGGAGATTTCACTGGT;

capgb-F: ACCTGGTGCTGGATAACAGG;

capgb-R: ATCTGAGCTTTGCCGTGTCT;

C5H11orf1-F: TGATGTCCAATAAAAGCCAGGT;

C5H110rf1-R: CACACATTGAGGCTCTGAAGT;

zdhhc16b-F: CCTGTGGAATTATGGGATGG;

zdhhc16b-R: ATGCTGCAGTGATGAGTTCG;

tekt3-F: AGATTTCAGCGCTGTCCGAT;

tekt3-R: AAGCAGCACGTTCACTCTGA;

mb-F: TGATCTGGTTCTGAAGTGCTG;

mb-R: GGCAAATCCGATCTCCTTGT;

s100s-F: CCAAGATGCCACGCTCAAAG;

s100s-R: CCCGCTAACACTTCTCTCGG.

\section{Morpholino-mediated gene knockdown}

The gene-specific morpholinos (MOs) were synthesized by the Gene Tools, LLC. For gene knockdown, the 2-3 $\mathrm{nL}$ of $0.3 \mathrm{mM}$ of gene-specific morpholinos were microinjected into the 1-2-cell-stage zebrafish embryos. Here, the morpholinos were used to block the splicing of the pre-mRNA, and therefore downregulate the target gene expression. The sequence of the morpholinos used in this study were as following:

capgb-MO: TCTGGAGGAACAAAGATGAGATGGT;

mb-MO: ATCAGAGAGTCCTGCTTTACCCTGA.

\section{Startle response test}

The startle response test in zebrafish was carried out following the procedure ${ }^{[40,41]}$. In this test, 20 zebrafish larvae at $5 \mathrm{dpf}$ were put into the culture dish for free swimming. When acoustic stimuli occured, 
the behaviour of the zebrafish was recorded by the high-speed camera (500 fps). In response to the stimuli, the zebrafish with normal auditory function would have a characteristic C-bend motion lasting less than $10 \mathrm{~ms}$, however, the zebrafish with damaged auditory function would not. Herein, both the distance moved and the number of the $\mathrm{C}$-bend motions were used to quantify the startle response.

\section{VOR test}

As previously described ${ }^{[41,42]}$, the VOR test was performed according to standard procedure. Briefly, the zebrafish larva at $5 \mathrm{dpf}$ was fixed in the chamber in a head-up position with $5 \%$ methylcellulose. The rotary platform, with the chamber unit fixed, rotated back and forth at a speed of $30 \mathrm{rpm}$. The eye movements of the zebrafish were recorded by infrared camera, and the periodical changes of the projection area of the eyes were used to evaluate the VOR.

\section{Imaging and statistical analysis}

For the confocal fluorescence microscopic analysis, the zebrafish were embedded in the $0.8 \%$ low melt agarose following anesthetization with MS-222, and the imaging was carried out by the Nikon A1 microscope. The Olympus MVX10 microscope was used for the bright-field imaging in the WISH experiments. All data were presented as mean with SD, and the unpaired Student's t-tests were used to determine statistical significance. A value of $P<0.05$ was considered statistically significant.

\section{Discussion}

In mammals, inner ear hair cells can detect the mechanical signals and transform them into physiological signals, which were then transmitted to the brain through the auditory neurons ${ }^{[43]}$. Inner ear hair cells can be divided into cochlear hair cells and vestibular hair cells, which play important roles in hearing and balance, respectively. The two types of hair cells differ from each other in both structure and function ${ }^{[7]}$.

As an excellent animal model, zebrafish have hundreds of hair cells, making it a good model to study the hair cell function. Similar to mammals, zebrafish have the inner ear, consisting of semicircular canals and otolith, however, zebrafish have no cochlea in their inner ear. Therefore, inner ear hair cells can be divided into macula hair cells and crista hair cells in zebrafish. Except for inner ear hair cells, zebrafish have a third type of hair cells, neuromast hair cells, in their lateral line system. The three different hair cells differ from each other in morphology, however, the molecular difference between them is unknown.

Here, we used single-cell RNA-sequencing method to analyze the gene expression pattern in zebrafish hair cells, and uncovered the molecular difference among the different types of hair cells. In brief, after quality control, the scRNA-seq data received dimensional reduction processing using the UMAP method, and all of the cells sequenced were classified as 21 clusters. After annotating according to the known marker genes and gene expression pattern in the database (http://zfin.org/), we identified four clusters of cells as macula hair cells, crista hair cells and neuromast hair cells. Further evidence confirmed our cell clustering and annotation in our following analysis. 
Knowing the different subtypes of hair cells, we performed a comparison among these hair cells, and found that the neuromast hair cells have more stronger expression of MET components and energy metabolism-related activity compared to the inner ear hair cells. Moreover, we analyzed the relationship between the known human NSHL genes and zebrafish hair cell marker genes revealed by the scRNA-seq, and found that $42.86 \%$ of human NSHL genes have orthologous genes expressed in zebrafish hair cells, which further reflects the reliability of our data. On the other hand, there are plenty of genes in our hair cell scRNA-seq gene pool that have not been reported to function in hair cells, which raises the possibility that these genes have potential value in hair cell function and even hearing loss gene identification. We randomly picked some of the marker genes for the further analysis, and found that these genes were specifically expressed in zebrafish inner ear or lateral line neuromast hair cells. Gene functional analysis also demonstrated that these genes were responsible for hair cell development or function, for that knockdown of the specific gene can lead to hair cell loss or hair cell dysfunction.

To sum up, in this study, we identified three subpopulations of hair cells, which corresponded with macula hair cells, crista hair cells and neuromast hair cells in zebrafish, through scRNA-seq. In addition, it also uncovered thousands of genes in zebrafish hair cells, which would be helpful for our research in hair cell biology in the future.

\section{Declarations}

\section{Ethics approval and consent to participate}

All zebrafish experimentation was carried out in accordance with the NIH Guidelines for the care and use of laboratory animals (http://oacu.od.nih.gov/regs/index.htm) and ethically approved by the Administration Committee of Experimental Animals, Jiangsu Province, China (Approval ID: 20180405039).

\section{Consent for publication}

The authors declare the consent for publication.

\section{Competing interests}

The authors declare that they have no conflicts of interest.

\section{Availability of data and materials}

All the high-throughput sequencing data generated in this study have been deposited in the GEO database under the accession number (It will be avliable before acceptance). All the experimental materials generated in this study are available from the corresponding authors upon reasonable request.

\section{Funding}


This work was supported in part by grants from National Natural Science Foundation of China, http://www.nsfc.gov.cn (2018YFA0801004 and 81870359 received by Dong Liu; 31900484 to Gangcai Xie); Natural Science Foundation of Jiangsu Province, http://kjjh.jspc.org.cn (BK20180048, and BRA2019278 received by Dong Liu; BK20190924 to Gangcai Xie; BK20190920 to Guanyun Wei). The funders had no role in study design, data collection and analysis, decision to publish, or preparation of the manuscript.

\section{Acknowledgements}

Not applicable.

\section{Author contributions}

Fuping Qian, Dong Liu, Gangcai Xie, and Renjie Chai conceived and designed the experiments, and wrote the manuscript. Fuping Qian, Xin Wang, Jie Gong, Chao Guo, Xiaoning Wang, Xu Zhang, Jinxiang Zhao, Cheng Wang, Mengting Xu, Yuebo Hu, Yajing Gao, and Guoli Yin performed the experiments. Fuping Qian, Guanyun Wei, Jiahui Kang, Gangcai Xie, Dong Liu and Renjie Chai analyzed the data. All authors read and approved the final manuscript.

\section{References}

1. Taylor, R.R., et al., Regenerating hair cells in vestibular sensory epithelia from humans. Elife, 2018. 7.

2. Kurima, K., et al., TMC1 and TMC2 Localize at the Site of Mechanotransduction in Mammalian Inner Ear Hair Cell Stereocilia. Cell Rep, 2015. 12(10): p. 1606-17.

3. Wagner, E.L. and J.B. Shin, Mechanisms of Hair Cell Damage and Repair. Trends Neurosci, 2019. 42(6): p. 414-424.

4. Nordang, L., et al., Glutamate is the afferent neurotransmitter in the human cochlea. Acta Otolaryngol, 2000. 120(3): p. 359-62.

5. Dalet, A., et al., Glutamate transporters EAAT4 and EAAT5 are expressed in vestibular hair cells and calyx endings. PLoS One, 2012. 7(9): p. e46261.

6. Highstein, S.M., et al., Evidence that protons act as neurotransmitters at vestibular hair cell-calyx afferent synapses. Proc Natl Acad Sci U S A, 2014. 111(14): p. 5421-6.

7. Dallos, P., The active cochlea. The Journal of Neuroscience, 1992. 12(12): p. 4575-4585.

8. Burns, J.C. and J.S. Stone, Development and regeneration of vestibular hair cells in mammals. Semin Cell Dev Biol, 2017. 65: p. 96-105.

9. Day, B.L. and R.C. Fitzpatrick, The vestibular system. Curr Biol, 2005. 15(15): p. R583-6.

10. Burns, J.C. and J.S. Stone, Development and regeneration of vestibular hair cells in mammals. Seminars in Cell \& Developmental Biology, 2017. 65: p. 96-105.

11. Patton, E.E., L.I. Zon, and D.M. Langenau, Zebrafish disease models in drug discovery: from preclinical modelling to clinical trials. Nat Rev Drug Discov, 2021(1474-1784 (Electronic)). 
12. Riley, B.B. and S.J. Moorman, Development of utricular otoliths, but not saccular otoliths, is necessary for vestibular function and survival in zebrafish. J Neurobiol, 2000. 43(4): p. 329-37.

13. Yao, Q., et al., Hearing Assessment in Zebrafish During the First Week Postfertilization. Zebrafish, 2016. 13(2): p. 79-86.

14. Smith, E.T., et al., Disruption of tmc1/2a/2b Genes in Zebrafish Reveals Subunit Requirements in Subtypes of Inner Ear Hair Cells. J Neurosci, 2020. 40(23): p. 4457-4468.

15. Pacentine, I.V. and T. Nicolson, Subunits of the mechano-electrical transduction channel, Tmc1/2b, require Tmie to localize in zebrafish sensory hair cells. PLoS Genet, 2019. 15(2): p. e1007635.

16. Erickson, T., et al., The Ihfp/5 Ohnologs Ihfp/5a and Ihfp/5b Are Required for Mechanotransduction in Distinct Populations of Sensory Hair Cells in Zebrafish. Front Mol Neurosci, 2019. 12: p. 320.

17. Navajas Acedo, J., et al., PCP and Wnt pathway components act in parallel during zebrafish mechanosensory hair cell orientation. Nat Commun, 2019. 10(1): p. 3993.

18. Lush, M.E., et al., scRNA-Seq reveals distinct stem cell populations that drive hair cell regeneration after loss of Fgf and Notch signaling. Elife, 2019. 8.

19. Kozlovskaja-Gumbriene, A., et al., Proliferation-independent regulation of organ size by Fgf/Notch signaling. Elife, 2017. 6.

20. Huo, L., et al., Single-cell multi-omics sequencing: application trends, COVID-19, data analysis issues and prospects. Brief Bioinform, 2021.

21. Xiao, T., et al., A GFP-based genetic screen reveals mutations that disrupt the architecture of the zebrafish retinotectal projection. Development, 2005. 132(13): p. 2955-67.

22. Yang, C.H., et al., Zona pellucida domain-containing protein $\beta$-tectorin is crucial for zebrafish proper inner ear development. PLoS One, 2011. 6(8): p. e23078.

23. Kozak, E.L., et al., Epithelial Planar Bipolarity Emerges from Notch-Mediated Asymmetric Inhibition of Emx2. Curr Biol, 2020. 30(6): p. 1142-1151 e6.

24. Pistocchi, A., et al., The zebrafish prospero homolog prox 1 is required for mechanosensory hair cell differentiation and functionality in the lateral line. BMC Dev Biol, 2009. 9: p. 58.

25. Aman, A. and T. Piotrowski, Wnt/beta-catenin and Fgf signaling control collective cell migration by restricting chemokine receptor expression. Dev Cell, 2008. 15(5): p. 749-61.

26. Seiler, C., et al., Myosin VI is required for structural integrity of the apical surface of sensory hair cells in zebrafish. Dev Biol, 2004. 272(2): p. 328-38.

27. Ernest, S. and F.M. Rosa, A genomic region encompassing a newly identified exon provides enhancing activity sufficient for normal myo7aa expression in zebrafish sensory hair cells. Dev Neurobiol, 2015. 75(9): p. 961-83.

28. Wu, Z. and U. Muller, Molecular Identity of the Mechanotransduction Channel in Hair Cells: Not Quiet There Yet. J Neurosci, 2016. 36(43): p. 10927-10934.

29. Qiu, X. and U. Muller, Mechanically Gated Ion Channels in Mammalian Hair Cells. Front Cell Neurosci, 2018. 12: p. 100. 
30. Xiong, W., et al., TMHS is an integral component of the mechanotransduction machinery of cochlear hair cells. Cell, 2012. 151(6): p. 1283-95.

31. Giese, A.P.J., et al., CIB2 interacts with TMC1 and TMC2 and is essential for mechanotransduction in auditory hair cells. Nat Commun, 2017. 8(1): p. 43.

32. Wang, Y., et al., Loss of CIB2 Causes Profound Hearing Loss and Abolishes Mechanoelectrical Transduction in Mice. Front Mol Neurosci, 2017. 10: p. 401.

33. Sollner, C., et al., Mutations in cadherin 23 affect tip links in zebrafish sensory hair cells. Nature, 2004. 428(6986): p. 955-9.

34. Sakaguchi, H., et al., Tip links in hair cells: molecular composition and role in hearing loss. Curr Opin Otolaryngol Head Neck Surg, 2009. 17(5): p. 388-93.

35. Choudhary, D., et al., Structural determinants of protocadherin-15 mechanics and function in hearing and balance perception. Proc Natl Acad Sci U S A, 2020. 117(40): p. 24837-24848.

36. Shi, J., et al., Spatio-temporal landscape of mouse epididymal cells and specific mitochondria-rich segments defined by large-scale single-cell RNA-seq. Cell Discov, 2021. 7(1): p. 34.

37. Yu, G., et al., clusterProfiler: an R package for comparing biological themes among gene clusters. OMICS, 2012. 16(5): p. 284-7.

38. Qian, F., et al., The slc4a2b gene is required for hair cell development in zebrafish. Aging (Albany NY), 2020. 12(19): p. 18804-18821.

39. Zhang, L., et al., THOC1 deficiency leads to late-onset nonsyndromic hearing loss through p53mediated hair cell apoptosis. PLoS Genet, 2020. 16(8): p. e1008953.

40. Wang, C., et al., Evaluation of the Hair Cell Regeneration in Zebrafish Larvae by Measuring and Quantifying the Startle Responses. Neural Plast, 2017. 2017: p. 8283075.

41. Gong, J., et al., Claudin h Is Essential for Hair Cell Morphogenesis and Auditory Function in Zebrafish. Front Cell Dev Biol, 2021. 9: p. 663995.

42. Sun, P., et al., An Assay for Systematically Quantifying the Vestibulo-Ocular Reflex to Assess Vestibular Function in Zebrafish Larvae. Front Cell Neurosci, 2018. 12: p. 257.

43. Zhang, S., et al., Characterization of Strip1 Expression in Mouse Cochlear Hair Cells. Front Genet, 2021. 12: p. 625867.

\section{Figures}

\section{Figure 1}

Single cell RNA-sequencing (scRNA-seq) of zebrafish hair cells. (A) The fluorescent image of the $\mathrm{Tg}$ (Brn3c:mGFP) transgenic zebrafish larva at $3 \mathrm{dpf}$. The GFP-expressing cells in the optic tectum, which were circled by the orange dotted line, are the retinal ganglion cells (RGCs), and the other GFP-expressing 
cells in the otic vesicle and lateral line system are hair cells. (B) The UMAP analysis of the zebrafish scRNA-seq data. The four clusters of cells circled by red dotted line were annotated as hair cells. (C-D) The number of cells and marker genes detected in each cluster. $(E)$ The dotplot of the top genes in each cluster.

\section{Figure 2}

The morphological feature of the three different types of hair cells in zebrafish. (A) The diagram of the zebrafish larva with hair cell labeling. UO, utricular otolith; SO, saccular otolith; UMHC, utricular macula hair cell; SMHC, saccular macula hair cell; ACHC, anterior crista hair cell; LCHC, lateral crista hair cell; PCHC, posterior crista hair cell. (B-D) The fluorescent image at high magnification of the three different types of hair cells. The diagrams of the different hair cells are presented in the boxes drawn with white dotted lines at bottom left. (E-F) The comparison of the kinocilia and the cell bodies of the three different

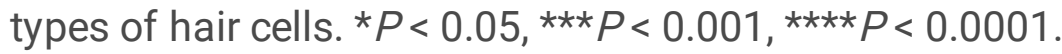

\section{Figure 3}

The hair cell classification in zebrafish. (A) The four clusters of hair cells can be further divided into three types, namely macula hair cells (cluster 5 ), crista hair cells (cluster 12), and neuromast hair cells (cluster 0 and 7). (B) The dotplot of top genes in the four clusters of hair cells. (C-F) The cnetplot of the genes expressed in the four clusters of hair cells. (G-I) The results of the WISH. The genes presented here expressed in the macula hair cells $(\mathbf{G})$, crista hair cells $(\mathbf{H})$, and neuromast hair cells $(\mathbf{I})$, respectively. The images at the bottom right in $(\mathbf{G})$ and $(\mathbf{H})$ shows the dorsal and enlarged view of the gene expression. The red arrowheads and arrows indicate the inner ear hair cells and neuromast hair cells, respectively.

\section{Figure 4}

The molecular difference among the different hair cells. (A) The marker genes expressed in different hair cells and the relationship among them. (B) The heatmap of the top marker genes in each cluster of hair cells. (C-E) The GO analysis reveals the different properties among the different hair cells in biological process (C), molecular function (D), and cellular component (E).

\section{Figure 5}


The distribution of the cells expressing the genes encoding the MET and tip link components. The feature plot and violin plot are showed for each gene.

\section{Figure 6}

The zebrafish hair cell scRNA-seq reveals potential hearing loss genes. (A) The relationship between the known human NSHL genes and zebrafish hair cell-enriched genes. (B) The list of the human NSHL genes, which have orthologs expressed in zebrafish hair cells. (C) Venn diagram of the zebrafish orthologs of human NSHL genes and marker genes expressed in each cluster of hair cells in zebrafish. The numbers indicate the number of the genes. (D) The expression pattern of the hair cell-enriched genes in zebrafish embryos revealed by WISH.

\section{Figure 7}

Functional analysis of the candidate genes involved in the hair cell development. (A-B) The capgb gene knockdown resulted in decreased neuromast hair cells in zebrafish. (C-D) The capgb-morphants showed less response to the acoustic stimuli. (E-F) The $m b$ gene knockdown resulted in reduced macula hair cells in zebrafish. (G-H) The $m b$-morphants showed damaged balance ability. ${ }^{\star} P<0.05,{ }^{\star} P<0.01, * \star \star P<$ $0.001, * \star \star \star P P 0.0001$.

\section{Supplementary Files}

This is a list of supplementary files associated with this preprint. Click to download.

- SupplementaryFigures.pdf 